Revue internationale d'éducation de Sèvres

\title{
Les performances du système éducatif marocain
}

\section{Zahra Zerrouqi}

\section{OpenEdition}

\section{Journals}

Édition électronique

URL : https://journals.openedition.org/ries/4474

DOI : $10.4000 /$ ries 4474

ISSN : 2261-4265

\section{Éditeur}

France Education international

\section{Édition imprimée}

Date de publication : 1 décembre 2015

Pagination : 22-28

ISSN : 1254-4590

\section{Référence électronique}

Zahra Zerrouqi, «Les performances du système éducatif marocain », Revue internationale d'éducation de Sèvres [En ligne], 70 | décembre 2015, mis en ligne le 18 janvier 2017, consulté le 01 juillet 2021. URL : http://journals.openedition.org/ries/4474 ; DOI : https://doi.org/10.4000/ries.4474

Ce document a été généré automatiquement le 1 juillet 2021.

(c) Tous droits réservés 


\title{
Les performances du système éducatif marocain
}

\author{
Zahra Zerrouqi
}

1 Avec un indice de développement de 0,591, le Maroc occupe la $130^{\mathrm{e}}$ place parmi les 177 pays pour lesquels l'IDH a été calculé en 2012. Parmi les principaux facteurs expliquant cette performance médiocre figurent les déficits significatifs en matière d'éducation. La situation critique du système d'éducation est soulignée par de nombreux rapports et études, aux plans national et international, et rend vitale une réforme radicale qui permettra au système éducatif marocain de progresser et de remplir ses missions.

D’après Mourshed et al., (2010), qui ont étudié vingt systèmes scolaires ayant progressé à travers le monde, tout système scolaire peut progresser de façon importante et rapidement (en moins de six ans), en mettant en place un ensemble de leviers prioritaires qui varient selon le niveau de performance dans lequel le système scolaire se situe (faible, correct, bon, très bon, excellent). C'est pourquoi il nous paraît primordial d'identifier d'abord les principaux facteurs de déclin du système, qui sont multiples et interdépendants. Dans cet article, l'accent est mis sur six facteurs clés : la faiblesse des acquisitions scolaires, l'incohérence linguistique, la pédagogie en difficulté, l'inadéquation des formations avec le marché du travail, le déficit de formation des enseignants et leurs conditions de travail difficiles et enfin l'image altérée de l'école. D'autres facteurs tels que l'analphabétisme, l'accès incomplet et inéquitable à l'enseignement, le redoublement et le décrochage scolaire, qui semblent être à la fois des facteurs et des résultats de l'échec de l'école marocaine, pourraient être évoqués. Cet article, au plan méthodologique, se fonde en grande partie sur l'analyse secondaire d'autres travaux scientifiques sur la même thématique.

\section{Faiblesse des acquisitions scolaires}

3 L'un des indicateurs les plus révélateurs de la qualité de l'enseignement / apprentissage apparaît à la lecture des résultats obtenus par le Maroc aux études TIMSS et PIRLS. Le 
TIMSS (Trends in International Mathematics and Science Study) et le PIRLS (Progress in International Reading Literacy Study) sont deux enquêtes organisées par l'IEA (International Association for the Evaluation of Educational Achievement). Le TIMSS compare les compétences des élèves en mathématiques et sciences en quatrième année de l'enseignement primaire et en huitième année, c'est-à-dire la deuxième année de l'enseignement collégial au Maroc. Le PIRLS compare les compétences en lecture et en compréhension des élèves de quatrième année de l'enseignement primaire. L'enquête TIMSS 2011 portant sur la $4^{\mathrm{e}}$ année du primaire indiquait un score global moyen de 335 en mathématiques et de 264 en sciences (tableau 1), très éloigné des scores de Singapour et de la Corée du Sud, en tête du classement.

Tableau 1. Scores globaux moyens et rang de cinq pays dont le Maroc en $4^{\mathrm{e}}$ année, selon l'étude TIMSS 2011

\begin{tabular}{|c|c|c|c|c|}
\hline & \multicolumn{2}{|c|}{ Mathématiques } & \multicolumn{2}{|c|}{ Sciences } \\
\hline & \multicolumn{2}{|c|}{$\begin{array}{l}4^{\mathrm{e}} \text { année } \\
\text { Moyenne internationale : } 500\end{array}$} & \multicolumn{2}{|c|}{$\begin{array}{l}4^{\mathrm{e}} \text { année } \\
\text { Moyenne internationale }: 500\end{array}$} \\
\hline & Score & $\begin{array}{l}\text { Rang } \\
\text { International/50 pays }\end{array}$ & Score & $\begin{array}{l}\text { Rang } \\
\text { International/50 pays }\end{array}$ \\
\hline Singapour & 606 & 1 & 587 & 1 \\
\hline Corée du Sud & 605 & 2 & 583 & 2 \\
\hline Finlande & 545 & 8 & 570 & 3 \\
\hline Tunisie & 359 & 47 & 346 & 48 \\
\hline Maroc & 335 & 49 & 264 & 49 \\
\hline Source : Provasnik & et al. (2012) & & & \\
\hline
\end{tabular}

4 Ainsi, en quatrième année de primaire, les résultats des élèves marocains en mathématiques et en sciences sont très en dessous de la moyenne internationale et occupent l'avant-dernière place au classement général.

5 En deuxième année du collège ( $8^{\mathrm{e}}$ année), les élèves marocains ont réalisé en mathématiques un score de 371 points, soit 129 points au-dessous de la moyenne internationale (tableau 2). En sciences, ils ont obtenu un score de 376 points, soit 124 points de moins que la moyenne internationale, occupant l'avant-dernière place. Singapour et la Corée du Sud figurent en tête du classement et les élèves tunisiens dépassent de 54 points en mathématiques et de 53 points en sciences les élèves marocains.

Tableau 2. Scores globaux moyens et rang de cinq pays dont le Maroc en $8^{e}$ année, selon l'étude TIMSS 2011

\begin{tabular}{|l|l|l|}
\hline & Mathématiques & Sciences \\
\hline
\end{tabular}




\begin{tabular}{|c|c|c|c|c|}
\hline & \multicolumn{2}{|c|}{$\begin{array}{l}8^{\mathrm{e}} \text { année } \\
\text { Moyenne internationale : } 500\end{array}$} & \multicolumn{2}{|c|}{$\begin{array}{l}8^{\mathrm{e}} \text { année } \\
\text { Moyenne internationale : } 500\end{array}$} \\
\hline & Score & $\begin{array}{l}\text { Rang } \\
\text { International/42 pays }\end{array}$ & Score & $\begin{array}{l}\text { Rang } \\
\text { International/42 pays }\end{array}$ \\
\hline Corée du Sud & 611 & 2 & 560 & 3 \\
\hline Singapour & 613 & 1 & 590 & 1 \\
\hline Finlande & 514 & 8 & 552 & 5 \\
\hline Tunisie & 425 & 30 & 439 & 29 \\
\hline Maroc & 371 & 40 & 376 & 41 \\
\hline Source: Provasn & $(20$ & & & \\
\hline
\end{tabular}

6 Dans l'étude PIRLS 2011, qui mesure la performance des apprenants en lecture en quatrième année du primaire, le Maroc occupait la dernière place avec un score de 310 points et un écart de -190 points sur la moyenne internationale (tableau 3 ). HongKong, la Finlande et Singapour occupent les trois premiers rangs.

Tableau 3. Scores moyens et rang de certains pays dont le Maroc, selon l'étude PIRLS 2011

\begin{tabular}{|l|l|l|}
\hline & \multicolumn{2}{|l|}{$\begin{array}{l}\text { e } \text { année } \\
\text { Moyenne internationale : } 500\end{array}$} \\
\cline { 2 - 3 } & Score & $\begin{array}{l}\text { Rang } \\
\text { International/45 pays }\end{array}$ \\
\hline Hong-Kong & 571 & 1 \\
\hline Finlande & 568 & 2 \\
\hline Singapour & 567 & 3 \\
\hline Maroc & 310 & 45 \\
\hline Source : Mullis et al. (2012) \\
\hline
\end{tabular}

7 Parmi les facteurs explicatifs de la faiblesse des acquis des élèves marocains avancés par le Conseil supérieur de l'éducation, de la formation et de la recherche scientifique (CSEFRS, 2014), figurent la non généralisation du pré-scolaire, le redoublement, les conditions d'apprentissage qu'offrent l'école, telles que les infrastructures, la taille des classes, les ressources pédagogiques et la charge horaire des enseignants. 


\section{Incohérence linguistique}

8 Dans le rapport qui clôture son mandat (1999-2004), la COSEF (Commission spéciale éducation-formation) a officiellement relié la "crise de l'école " à l'incohérence linguistique scolaire. L'arabisation n'a pas atteint l'enseignement supérieur, si bien qu'un élève titulaire d'un baccalauréat en arabe et qui veut poursuivre ses études scientifiques à l'université doit maîtriser le français. Par ailleurs, l'arabisation, qui était censée réduire les inégalités sociales en remplaçant le français par l'arabe à l'école marocaine, s'est transformée en un processus de sélection sociale. Errihani (2008) évalue la politique d'arabisation au Maroc comme une stratégie idéologique mise en place par les élites pour verrouiller l'accès à la formation qui produit les élites du pays. Devant cette situation, les jeunes marocains adoptent une attitude plutôt hostile à l'égard de l'arabisation. Les élèves marocains font aujourd'hui preuve d'un usage déficitaire de l'arabe, leur langue officielle, et du français, leur première langue étrangère. Cette faiblesse des acquis linguistiques est soulignée par les enseignants et par les acteurs extérieurs au système. Cette situation est inquiétante, lorsqu'on sait que la non maîtrise des langues étrangères constitue un obstacle à l'insertion professionnelle, dans la mesure où une majorité de postes, notamment dans le secteur privé, requiert la maîtrise de l'arabe et d'au moins une langue étrangère. Cette non maîtrise pénalise nombre d'étudiants et favorise ceux, généralement de milieu plus aisé, qui bénéficient d'un enseignement de langues étrangères conséquent. Cette crise linguistique perdure malgré les recommandations des deux dernières réformes du système éducatif marocain, à savoir la Charte nationale de l'éducation et de la formation (1999-2009) et le Plan d'urgence (2009-2012). Notons qu'actuellement, la politique linguistique se trouve confrontée à des choix dictés par des considérations idéologiques et par des conflits politiques opposant différents courants de pensée : les partisans de l'arabisation, les promoteurs de la francophonie, les partisans de l'arabe dialectal et les représentants du mouvement amazigh. Par ailleurs, de nombreux Marocains voient aujourd'hui l'utilité de l'anglais, qui leur offre un meilleur accès à l'information, à la recherche et au marché du travail et demandent que cette langue occupe la place de première langue étrangère au Maroc, au lieu du français.

\section{Une pédagogie en difficulté}

9 Depuis 2000, tous les cycles de l'enseignement scolaire ont enregistré de nombreuses avancées sur le plan pédagogique: les curricula ont été révisés et de nouvelles orientations pédagogiques ont été -adoptées, telles que l'approche par compétences (APC). De même, les méthodes d'évaluation et l'organisation des examens ont été revues. Cependant, sur le terrain, ces initiatives n'ont pas produit d'amélioration substantielle des résultats des apprenants et de l'acte d'enseignement au sein de la classe, en raison de plusieurs blocages. En effet, l'APC définit les compétences attendues des élèves à certains moments de leurs cursus scolaire mais reste discrète sur la manière de les rendre compétents. En outre, la complexité et l'exigence théorique de l'APC sont très éloignées des conditions concrètes de sa mise en œuvre. Ainsi, les conditions minimales en matière d'infrastructure, d'effectifs des élèves par classe et de formation des enseignants ne sont pas encore généralisées, ce qui limite fortement l'application de l'APC (AFD, 2010). Dans le cadre du Programme d'urgence (2009-2012), 
le Département de l'éducation nationale avait adopté la pédagogie de l'intégration comme cadre méthodologique en vue d'opérationnaliser l'APC. Cependant, la mise en œuvre de cette pédagogie a rencontré de sérieuses difficultés, qui ont conduit le Département à arrêter sa généralisation en 2012 (CSEFRS, 2014).

Par ailleurs, les orientations pédagogiques sont davantage portées par les organisations internationales que discutées par les mouvements pédagogiques nationaux. Les contenus sont parfois même directement inspirés des manuels européens. Une telle situation rend difficile voire improbable leur appropriation par les acteurs de terrain que sont les enseignants, renforce la réalité de dépendance dans laquelle baigne le système éducatif marocain, et pourrait emprisonner des générations dans des théories qui peuvent être dépassées en silence au sein du mouvement de la société occidentale.

11 En termes d'évaluation, les révisions ont accordé plus de place au contrôle continu dans l'évaluation des élèves à tous les niveaux, ce qui engendre des situations de gonflement des notes dans l'école publique et plus particulièrement dans le secteur privé, marqué par des différences flagrantes entre la moyenne des contrôles continus et la moyenne de l'examen régional et national au baccalauréat. Cette situation permet d'élever les seuils de présélection aux concours, pénalisant ainsi les élèves méritants. De plus, l'adoption des systèmes d'examen régional/national dans le secondaire entraîne une marginalisation par les élèves de certaines matières non incluses dans les épreuves certificatives.

12 En outre, on néglige la dimension temporelle dans le processus enseignement/ apprentissage, à savoir la répartition horaire des matières enseignées et le nombre d'heures de travail des enseignants assez élevé, surtout dans le primaire.

\section{Inadéquation des formations avec le marché du travail}

13 Selon les statistiques du Haut Commissariat au plan (HCP, 2014), le taux de chômage augmente avec l'élévation du niveau d'instruction et ceci pour les différentes tranches d'âge (15-24 ans, 25-34 ans et 35-44 ans). En effet, le taux de chômage s'élève à 62,7 \% pour les demandeurs d'emploi de niveau supérieur de la tranche d'âge 15-24 ans.

Le chômage des diplômés de certaines filières universitaires à fort effectif, d'une part, et les pénuries de compétences dans certains segments, d'autre part, indiquent que le système d'éducation marocain ne transmet pas aux étudiants les compétences nécessaires à une insertion professionnelle réussie. Ainsi s'installe un décalage entre, d'un côté, les attentes des acteurs et de la collectivité et, de l'autre, la qualité de la réponse apportée par le système d'éducation/formation, qui commence à montrer des signes prononcés d'inadéquation au regard des mutations qui touchent l'économie marocaine et l'ensemble de la vie sociale du pays.

\section{Les enseignants : déficit de formation et conditions de travail difficiles}

Malgré le recrutement annuel de larges effectifs d'enseignants, les besoins croissent, compte tenu de la généralisation progressive de l'enseignement et des départs à la retraite. 

freine l'amélioration de la qualité de l'enseignement et conduit à un réel décalage entre les nouvelles orientations et les pratiques pédagogiques en classe. En effet, le mode de recrutement en vigueur dans les Centres régionaux des métiers d'éducation et de formation (CRMEF) dont les missions portent, entre autres, sur la qualification des cadres enseignants stagiaires, connaît certaines limites, dont l'hétérogénéité des profils des stagiaires issus de différentes spécialités des universités (biologie, physique, lettres, sciences économiques et juridiques, etc.) et qui sont amenés à exercer dans le cycle primaire. Cela rend difficile leur qualification professionnelle et compromet la qualité de leurs pratiques enseignantes. En outre, on note l'absence d'une structure dédiée à la formation des formateurs, ce qui se répercute négativement sur la qualité de l'encadrement au sein de ces CRMEF. L'accès au métier est une étape clé dans la carrière de l'enseignant et requiert une attention toute particulière. Au Maroc, l'organisation de la formation initiale, son contenu et sa durée dépendent du statut du centre de formation, alors qu'au niveau international, la tendance converge vers un rattachement au système universitaire. L'intégration des centres dans le système universitaire pourrait être bénéfique à plus d'un titre : élargissement du champ des connaissances, augmentation du volume de l'aspect pratique, développement de l'esprit critique, initiation à la recherche en matière d'éducation, renforcement de la régionalisation de la gestion des ressources, etc. Au cours de l'exercice de leur métier, les enseignants ne bénéficient pas d'une formation continue planifiée, visant leur recyclage et leur mise à niveau au sujet des développements récents de leur métier (approches pédagogiques, nouvelles technologies d'information, qualité, etc.). De plus, cette formation continue est plutôt régie par une logique de consommation des crédits, au détriment de la satisfaction des besoins réels des enseignants (CSEFRS, 2014).

Par ailleurs, les conditions de travail difficiles constituent un obstacle à l'amélioration de la qualité de l'enseignement. Elles résultent de différentes causes: surcharge de travail due à des classes pléthoriques ou à des niveaux multiples, insuffisance des moyens pédagogiques, infrastructure parfois précaire de l'école, notamment en milieu rural. À cela s'ajoute le sentiment d'injustice ressenti particulièrement par les enseignants du primaire travaillant dans des zones dépourvues de simples moyens de vie tels que l'eau potable, l'électricité, le réseau téléphonique et les moyens de transport, et qui sont éloignés de leurs familles pour une longue période. Dans le milieu rural, la condition sociale des élèves constitue également un obstacle au bon déroulement de l'apprentissage.

\section{Image altérée de l'école}

La confiance de la société envers l'école s'est bien altérée ces dernières décennies. Ce phénomène trouve son explication d'une part dans l'élévation du taux de chômage chez les diplômés, relativisant ainsi le rôle de l'éducation en tant que moyen de l'ascension sociale, et d'autre part dans l'image de l'environnement scolaire, qui est affectée par des phénomènes d'incivisme. En effet, selon les données TIMSS et PIRLS pour la période 2001-2011, plus de $60 \%$ des élèves marocains en quatrième année du primaire et $49 \%$ en deuxième année collégiale sont scolarisés dans des écoles où des problèmes de discipline tels que l'absentéisme, le retard, la tricherie, le vandalisme, le vol et la 
violence verbale et physique envers les enseignants sont fortement présents (CSEFRS, 2014). En outre, les infrastructures délabrées continuent à altérer l'image de l'école.

Ainsi, le système d'éducation/formation marocain, qui peine à inculquer les apprentissages, peine aussi à éduquer. L'enseignement dont on a besoin aujourd'hui devrait être vecteur de transmission des valeurs civiques et éthiques qui cultivent une citoyenneté responsable et entretiennent le désir de contribuer au bien-être commun. Or l'école marocaine semble ne transmettre que faiblement les valeurs de droit, de citoyenneté et de respect indispensables à la bonne intégration sociale, comme en témoigne la multiplication des incivilités telles que les faits de violences et de dégradation des biens, ou encore la banalisation de la fraude aux examens.

Les facteurs responsables du déclin du système éducatif marocain sont pour la plupart interdépendants. En effet, d'une part, le déficit de formation des enseignants et leurs conditions de travail difficiles, la pédagogie en difficulté et l'incohérence linguistique contribuent à la faiblesse des acquisitions scolaires. D'autre part, l'inadéquation des formations avec le marché du travail et le manque de civilité altèrent l'image de l'école. Dans ces conditions, comment le système éducatif marocain pourrait-il progresser ? D'après Mourshed et al. (2010), la première étape est d'identifier le niveau de performance du système éducatif. Dans le cas du Maroc, le système éducatif présente un niveau de performance faible et doit progresser vers le niveau correct, avant de passer aux niveaux bon, très bon puis excellent. Les efforts doivent se focaliser sur la mise en place des bases de l'apprentissage de la langue et des mathématiques, particulièrement au cycle primaire. Par ailleurs, la formation initiale des enseignants et leur motivation, le développement des compétences nécessaires à l'insertion professionnelle ainsi que l'amélioration de l'image de l'école sont des facteurs incontournables de l'amélioration du niveau de compétence de tout système scolaire.

\section{BIBLIOGRAPHIE}

AFD : Agence française de développement (2010), Les réformes curriculaires par l'approche par compétences en Afrique, -document de travail $n^{\circ} 97$.

Conseil supérieur de l'éducation, de la formation et de la recherche scientifique : CSEFRS (2014) : La mise en cuvre de la charte nationale d'éducation et de formation 2000-2013, acquis, déficits et défis, rapport analytique.

ERRIHANI M. (2008): Language policy in Morocco: implications of recognizing and teaching Berber, Saarbrucken: VDM Verlag.

Haut Commissariat au plan : HCP (2014) : Activité, emploi et chômage, premiers résultats. [http:// www.hcp.ma]

MOURSHED M., CHIJIOKE C., BARBER M. (2010) : How the world's most improved school systems keep getting better, McKinsey\&Company. 
MULLIS I.V.S., MARTIN M.O., FOY P., DRUCKER K.T. (2012) : PIRLS 2011 International Results in Reading, Chestnut Hill, M.A., Boston College.

PROVASNIK S., KASTBERG D., FERRARO D., LEMANSKI N., ROEY S., JENKINS F. (2012) : Highlights From TIMSS 2011 : Mathematics and Science Achievement of U.S. Fourth- and Eighth-Grade Students in an International Context (NCES 2013-009 Revised). National Center for Education Statistics, Institute of Education Sciences, U.S. Department of Education, Washington DC.

\section{INDEX}

Mots-clés : enseignant, politique linguistique, système éducatif, relation formation-emploi Index géographique : Maroc

Keywords : teachers, educational system, language policy, training-employment relationship Palabras claves : docente, sistema educativo, política lingüística, relación formación-empleo

\section{AUTEUR}

\section{ZAHRA ZERROUQI}

Zahra Zerrouqi est docteur en sciences de l'environnement de l'Université Mohammed Premier, Oujda (Maroc). Elle a d'abord enseigné dans le secondaire avant de rejoindre le Centre régional des métiers de l'éducation et de formation (CRMEF) de l'oriental, en tant qu'enseignantechercheuse intervenant dans la formation et l'encadrement des enseignants stagiaires. Courriel : zzerrouqi@yahoo.fr 